\title{
Prevalence and Associated Factors of Uraemic Pruritus in Continuous Ambulatory Peritoneal Dialysis Patients
}

\author{
Jianying Li, Qunying Guo, Jianxiong Lin, Chunyan Yi, Xiao Yang and Xueqing Yu
}

\begin{abstract}
Objective Uraemic pruritus is a distressing symptom that has a negative impact on the quality of life for dialysis patients. The pathophysiology of pruritus in peritoneal dialysis (PD) patients is still poorly understood. The present study aims to investigate the prevalence and related risk factors of pruritus in continuous ambulatory peritoneal dialysis (CAPD) patients.

Methods In total, 362 CAPD cases were investigated from January 2012 to April 2013. Pruritus was assessed by visual analogue scale.

Results The prevalence of severe pruritus and mild to moderate pruritus was $12.7 \%$ and $52.5 \%$, respectively. The patients with severe pruritus had the longest duration of PD $(\mathrm{p}<0.001)$, Pittsburgh Sleep Quality Index (PSQI) score $(\mathrm{p}<0.001)$, Beck Depression Inventory (BDI) score $(\mathrm{p}=0.003)$, intact parathyroid hormone (iPTH) level ( $\mathrm{p}=0.009)$, and the lowest Medical Outcomes Short Form 36 Health Survey, Physical Component Score (SF-36 PCS) $(\mathrm{p}<0.001)$ among the three groups. The patients with mild to moderate pruritus had a significantly higher $\mathrm{iPTH}$ level $(\mathrm{p}=0.004)$ compared with the patients without pruritus. A multivariate logistic regression for pruritus showed that higher PSQI score [odds ratio $(\mathrm{OR})=1.305, \mathrm{p}=0.001$ ], higher BDI score $(\mathrm{OR}=1.429, \mathrm{p}=0.002)$, longer vintage $(\mathrm{OR}=1.039, \mathrm{p}=0.004)$, and higher iPTH level $(\mathrm{OR}=1.317, \mathrm{p}=$ $0.014)$ were independently associated with pruritus.

Conclusion The prevalence of uraemic pruritus was $65.2 \%$ in CAPD patients. Sleep disorder, depression, longer vintage, and a higher iPTH level were independent associated factors for pruritus in CAPD patients.
\end{abstract}

Key words: peritoneal dialysis, pruritus, depression, sleep disorder, quality of life

(Intern Med 54: 2827-2833, 2015)

(DOI: 10.2169/internalmedicine.54.4516)

\section{Introduction}

Uraemic pruritus is a distressing symptom that has a negative impact on the quality of life (QoL) for dialysis patients (1). The prevalence of pruritus ranges from 63 to $84 \%$ in dialysis patients $(2,3)$. Although xerosis, alterations of mineral metabolism (4), iron deficiency anemia, hepatitis $\mathrm{C}$ virus (HCV) infection, and inflammation were reported to be causal mechanisms of prutirus (5-7), the pathophysiology of pruritus in peritoneal dialysis (PD) patients is still poorly understood.

Recent studies also demonstrated strong associations of severe pruritus with depression symptoms and poorer sleep quality in hemodialysis (HD) patients (8-10). The Dialysis
Outcomes and Practice Patterns Study (DOPPS) reported that HD patients with severe pruritus were more likely to have poor sleep quality and depression. Vandana et al. also found significant associations among itching intensity and severity and sleep. However, few studies have assessed the related mechanisms of pruritus and its role in sleep disorder and depression in PD patients. Thus, the present study aimed to assess the prevalence of pruritus, explore the associated factors of the condition, and understand its interrelationship between mineral metabolism, sleep quality, and depression in a cohort of continuous ambulatory peritoneal dialysis (CAPD) patients. 


\section{Materials and Methods}

\section{Objectives}

This was a single-center, cross-sectional, and observational study. The present study aimed to investigate the prevalence and related risk factors of pruritus in CAPD patients.

\section{Participants}

The inclusion criteria were as follows, patients who: 1) had received CAPD for more than 3 months; 2) are older than 18 years of age; 3 ) can provide a signed informed consent form. The exclusion criteria were as follows, patients who: 1) have a presence of malignancy and active infection; 2) have required an operation within the prior month; 3) have had a recent hospitalization within 1 month; 4) have a psychotic disorder or dementia; 5) have acute hepatitis or cholestatic liver disease, or primary skin disorder; 6) have previously received HD or renal transplantation before PD. The data for demographic information, clinical data, and record of skin problems were collected.

\section{Ethics}

The study protocol was approved by the Ethics Committee of The First Affiliated Hospital at Sun Yat-sen University. The PD patients were investigated from January 2012 to April 2013 at our PD patients' clinic. Only the patients who agreed to participate in this study and signed the informed consent form were enrolled. The printed questionnaire in Chinese, which includes the visual analogue scale, sleep quality survey, Medical Outcomes Short Form 36 Health Survey, and Beck Depression Inventory-II, was completed by the patients during the visit.

\section{Pruritus treatment and assessment}

The severity of pruritus was assessed by the visual analogue scale (VAS), which ranged from 0 to $10(0=$ no pruritus to $10=$ intolerable pruritus) as described in a previous study (6). Each patient completed the VAS with the help of study nurses. According to their VAS scores, the patients were divided into three groups: no pruritus was defined as VAS scores $=0$ (group 1); mild to moderate pruritus was defined as VAS scores $=1-5$ (group 2); and severe pruritus was defined as VAS scores $>5$ (group 3). Of the patients, 16.4 percent of them received oral antihistamine for pruritus, as needed.

\section{Assessment of sleep quality}

The Chinese version of the Pittsburgh Sleep Quality Index (PSQI) had been validated for sleep quality for Chinese dialysis patients (11). It contains 19 questions and yields a global PSQI score ranging from 0 to 21. Patients with PSQI scores $\geq 5$ were considered to be "poor sleepers" and those with a score of $<5$ were considered to be "good sleepers".

\section{Assessment of quality of life}

The QoL was measured by the Medical Outcomes Short Form 36 Health Survey (SF-36), which has been validated for the Chinese population and for patients receiving dialysis treatment (12). This was a short-form measure of a patient's general health status, which included a physical component score (PCS) and a mental component score (MCS). A higher score indicates a better QoL.

\section{Assessment of depression}

The Beck Depression Inventory-II (BDI-II) was used for an assessment of depression in CAPD patients. Depression was defined as a BDI-II score $\geq 14$, which was based on a diagnosis standard that is used for dialysis patients (13).

\section{Assessment of malnutrition-inflammation status}

The malnutrition-inflammation score (MIS) included the 7-component conventional subjective global assessment (SGA) of nutrition and 3 other elements [body mass index (BMI), serum albumin, and serum transferrin]. There are 4 levels of severity, ranging from 0 (normal) to 3 (very severe) for each MIS component (11).

\section{Assessment of comorbidity}

Each patient's comorbidity score was calculated according to the Charlson Comorbidity Index (CCI), by assigning a weight of 2 to diabetes, stroke, renal insufficiency, and malignancy and a weight of 1 to the other comorbidities (14). Twelve patients had myocardial infarctions, 62 patients had congestive heart failure, 39 patients suffered from peripheral atherothrombotic vascular disease, and 12 patients had cerebrovascular disease. Five patients had chronic lung disease, 4 were diagnosed with moderate or severe liver illness, 3 suffered from gastrointestinal ulcer, 4 had hemiplegic paralysis, and 3 patients had a malignant tumor.

\section{Statistical analyses}

The patients' characteristics were presented as mean \pm SD for continuous variables, and showed as the median (1st and 3rd quartile) for abnormally distributed continuous variables. Categorical variables were shown as percentages. A one-way analysis of variance (ANOVA) test was used for normally distributed variables among the three groups. A comparison of abnormally distributed continuous variables was performed using a non-parametric Kruskal-Wallis test. For categorical variables, a Chi-square test was used. A post hoc Dunnett's test was used to compare groups 1 and 2, group 1 and 3, and group 2 and 3.

Sex, age, time of dialysis, diabetes, CCI, and factors that reached statistical significance were selected for further multivariable analyses. Logistic regressions and multivariate linear regressions were performed for a multivariable-adjusted analysis to explore the predictors of pruritus, depression, sleep disorder and QoL. All calculations were performed with SPSS 13.0 (Statistical Product and Service Solutions, 
Chicago, USA). A p value of less than 0.05 was considered to be significant.

\section{Results}

A total of 516 patients were followed regularly at the clinic from January 2012 to April 2013. Due to the exclusion criteria, 62 patients were excluded. Ninety-two patients declined to participate in this study. In the end, 362 PD patients were recruited.

\section{Patient demographics}

A total of 362 CAPD patients $(53.6 \%$ men, mean age $49.9 \pm 16.7$ years) were observed, with a median PD duration of 16 months. The etiology of End-Stage Renal Disease (ESRD) was chronic glomerulonephritis in 193 cases (53.4\%), hypertensive nephrosclerosis in 37 cases (10.2\%), diabetic nephropathy in 100 cases $(27.7 \%)$, polycystic kidney disease in 8 cases $(2.2 \%)$, and unknown causes in 24 cases $(6.5 \%)$.

\section{Prevalence of pruritus}

The prevalence of severe, mild to moderate pruritus, and no pruritus was $12.7 \%, 52.5 \%$, and $34.8 \%$, respectively.

\section{Characteristics of CAPD patients with pruritus}

Among the three groups, the patients with severe pruritus had the longest duration of PD $(\mathrm{p}<0.001)$, the highest diabetes mellitus proportion $(\mathrm{p}=0.013)$, PSQI score $(\mathrm{p}<0.001)$, BDI score $(\mathrm{p}=0.003)$, MIS score $(\mathrm{p}=0.013)$, and intact parathyroid hormone $(\mathrm{iPTH})$ level $(\mathrm{p}=0.009)$ and the lowest SF36 PCS $(\mathrm{p}<0.001)$. Furthermore, the patients with mild to moderate pruritus had a significantly higher iPTH level $(\mathrm{p}=$ 0.004) compared with the patients without pruritus (as shown in Table 1, Fig. 1, 2).

\section{Multivariate logistic regression analysis for pruritus, sleep disorder, and depression in CAPD patients}

The multivariable-adjusted logistic regression model showed that the PSQI $(\mathrm{OR}=1.305, \mathrm{p}=0.001)$, BDI $(\mathrm{OR}=$ 1.429, $\mathrm{p}=0.002)$, time of $\mathrm{PD}(\mathrm{OR}=1.039, \mathrm{p}=0.004)$, and iPTH level $(\mathrm{OR}=1.317, \mathrm{p}=0.014)$ were independent predictors for pruritus after adjusting for age, sex, diabetes, PD duration and season. Pruritus $(\mathrm{OR}=1.653, \mathrm{p}=0.01)$, BDI $(\mathrm{OR}=1.065, \mathrm{p}=0.024)$, and MIS score $(\mathrm{OR}=1.186, \mathrm{p}=0.014)$ were independent predictors for sleep disorder after adjusting for age, sex, PD duration, diabetes and CCI. The variables female $(\mathrm{OR}=6.458, \mathrm{p}=0.013)$, diabetes $(\mathrm{OR}=1.83, \mathrm{p}=$ 0.004), and PSQI (OR=1.171, p=0.018) were independent predictors for depression after adjusting for age, sex, PD duration, diabetes and CCI (as shown in Table 2).

\section{Multivariate linear regression analysis for QOL in CAPD patients}

In a multiple linear regression analysis, the BDI $(\beta=-0.31$, $\mathrm{p}=0.001)$, MIS score $(\beta=-0.26, \mathrm{p}=0.001)$, age $(\beta=-0.25, \mathrm{p}=$
$0.001)$, and higher calcium $\times$ phosphate product $(\beta=-0.15$, $\mathrm{p}=0.019)$ were independent predictors for PCS. In addition, sleep disorder $(\beta=-0.27, \mathrm{p}=0.001)$, depression $(\beta=-0.40, \mathrm{p}=$ $0.001)$, and age $(\beta=0.18, p=0.024)$ were independent predictors for MCS (as shown in Table 3 ).

\section{Discussion}

In this present study, the prevalence of severe pruritus and mild to moderate pruritus was 12.7 , and $52.5 \%$, respectively. Sleep disorder, depression, longer PD duration, and higher iPTH level were independent predictors for pruritus in CAPD patients. After controlling for potential confounding factors, significant associations among uremic pruritus, sleep quality, and depression were demonstrated in CAPD patients.

We demonstrated bidirectional associations existing between pruritus and sleep quality in CAPD patients. Pruritus was shown to be an independent risk factor for sleep disorder, while sleep quality was also shown to be an independent predictor for pruritus in this study. Indeed, severe uremic pruritus have been reported to occur more often at night (15). Patients with extreme pruritus had a 2.3 to 4.1 times greater adjusted odds ratio of not having enough sleep, either being sleepy during the day or being awake at night (16). Thus, DOPPS suggested that pruritus was associated with a higher mortality risk due to pruritus-induced sleep disturbance in HD patients (8). Moreover, the presence of sleep disorder at night may affect pruritus perception. Previous studies suggested that a dysfunction of the circadian rhythm, involving the release of itch mediators, was associated with the aggravation of pruritus (17), which indicated sleep disturbances were associated to the development of severe pruritus. Therefore, in addition to the previous unidirectional speculation that pruritus causes sleep disturbances in HD patients, results from the present study revealed a bidirectional association between pruritus and sleep disturbances in CAPD patients.

Another interesting finding of this study was that depression was also an independent risk factor for pruritus. Previous studies reported an increased sensitivity to aversive stimuli due to depressive symptom-inducing chemical changes in serotonergic or noradrenergic function (18), which suggested that depressive symptoms might worsen pruritus. Another longitudinal analytic study suggested that patients with depressive symptoms were likely to show a future risk of severe pruritus, furthermore, depressive symptom-induced sleep disturbances were also possibly associated to the development of severe pruritus (19). These reports were in agreement with our results suggesting an association between pruritus and depressive symptoms. Moreover, both pruritus and depression were the bidirectional factors of sleep quality in this study. Several studies have reported the relationship between depressive symptoms and subsequent development of sleep disturbances $(20,21)$. These findings supported the presumption that sleep distur- 
Table 1. Clinical, Demographic and Biochemical Characteristics in 362 CAPD Patients with and without Pruritus.

\begin{tabular}{|c|c|c|c|c|c|}
\hline & $\begin{array}{l}\text { All patients } \\
(\mathrm{n}=362)\end{array}$ & $\begin{array}{l}\text { No pruritus } \\
(\mathrm{n}=126)\end{array}$ & $\begin{array}{l}\text { Mild to moderate } \\
\text { pruritus }(\mathrm{n}=190)\end{array}$ & $\begin{array}{l}\text { Severe pruritus } \\
\quad(\mathrm{n}=46)\end{array}$ & $\mathrm{p}$ value \\
\hline Male (\%) & 53.6 & 51.5 & 56.6 & 51.2 & 0.773 \\
\hline Age (year) & $49.9 \pm 16.7$ & $47.6 \pm 15.1$ & $48.8 \pm 15.9$ & $58.8 \pm 14.3^{\mathbf{a}, \mathbf{b}}$ & $0.007^{*}$ \\
\hline Diabetes (\%) & 27.7 & 15.7 & 34.1 & $34.8^{\mathbf{a}}$ & $0.013^{*}$ \\
\hline Vintage (months) & $16(10,29.7)$ & $13.5(8,22.5)$ & $18(11,31)$ & $23(12.5,48)^{\mathrm{a}}$ & $0.001 *$ \\
\hline Occupational status $1 / 2^{\#}$ & $208 / 154$ & $35 / 91$ & $58 / 132$ & $8 / 38$ & 0.115 \\
\hline PSQI & $9.4 \pm 4.1$ & $7.7 \pm 5.3$ & $9.3 \pm 4.2$ & $12.3 \pm 5.6^{\mathbf{a}, \mathbf{b}}$ & $<0.001 * *$ \\
\hline BDI score & $10(5,18.8)$ & $10.5(4,19.3)$ & $8(4,13)$ & $17(6,22)^{\mathbf{a}, \mathbf{b}}$ & $0.003 *$ \\
\hline MIS score & $4.5 \pm 2.7$ & $4.1 \pm 2.4$ & $4.4 \pm 2.4$ & $5.9 \pm 3.7^{\mathbf{a}, \mathbf{b}}$ & $0.013^{*}$ \\
\hline $\mathrm{CCI}$ & $4.6 \pm 1.9$ & $4.4 \pm 1.9$ & $4.2 \pm 1.6$ & $5.8 \pm 2.3$ & 0.102 \\
\hline PCS & $40.7 \pm 7.8$ & $41.2 \pm 7.5$ & $42.5 \pm 6.4$ & $36.0 \pm 9.3^{\mathbf{a}, \mathbf{b}}$ & $<0.001 * *$ \\
\hline MCS & $44 \pm 9.8$ & $45.2 \pm 9.7$ & $43.7 \pm 10.2$ & $42.9 \pm 9.7$ & 0.454 \\
\hline BMI & $22.3 \pm 3.1$ & $22.7 \pm 2.8$ & $21.9 \pm 3.3$ & $22.5 \pm 2.9$ & 0.399 \\
\hline Dialysis dose (L/Day) & $8.4 \pm 0.5$ & $8.2 \pm 0.4$ & $8.6 \pm 0.4$ & $8.6 \pm 0.3$ & 0.519 \\
\hline Season $(\%)$ & & & & & 0.248 \\
\hline Spring & 25.5 & $30.9(39 / 126)$ & $21(40 / 190)$ & $29.2(13 / 46)$ & \\
\hline Summer & 32.8 & $33.8(42 / 126)$ & $20(38 / 190)$ & $12.5(6 / 46)$ & \\
\hline Autumn & 18.2 & $14.7(19 / 126)$ & $28(53 / 190)$ & $20.8(10 / 46)$ & \\
\hline Winter & 23.4 & $20.6(26 / 126)$ & $31(59 / 190)$ & $37.5(17 / 46)$ & \\
\hline hsCRP (mg/L) & $1.8(0.8,6.6)$ & $1.4(0.5,6.6)$ & $1.9(0.9,4.8)$ & $2.6(1.3,10.9)$ & 0.084 \\
\hline $\mathrm{Hb}(\mathrm{g} / \mathrm{dL})$ & $106.8 \pm 18.6$ & $105.9 \pm 21.5$ & $107.8 \pm 16.5$ & $105.2 \pm 18.1$ & 0.68 \\
\hline Creatinine (umol/L) & $936.2 \pm 330.2$ & $904.6 \pm 294.1$ & $947.6 \pm 323.3$ & $962.1 \pm 396.7$ & 0.622 \\
\hline Albumin $(\mathrm{g} / \mathrm{L})$ & $38.1 \pm 4.2$ & $38.6 \pm 4.0$ & $38.4 \pm 4.2$ & $36.5 \pm 4.2$ & 0.087 \\
\hline Prealbumin (g/L) & $337.9 \pm 82.9$ & $348.8 \pm 79$ & $337.1 \pm 88.9$ & $312.1 \pm 61.8$ & 0.179 \\
\hline Transferrin (g/L) & $2.3 \pm 0.5$ & $2.4 \pm 0.5$ & $2.3 \pm 0.5$ & $2.2 \pm 0.4$ & 0.152 \\
\hline Calcium $(\mathrm{mmol} / \mathrm{L})$ & $2.4 \pm 0.2$ & $2.3 \pm 0.2$ & $2.4 \pm 0.2$ & $2.4 \pm 0.3$ & 0.14 \\
\hline Phosphate $(\mathrm{mmol} / \mathrm{L})$ & $1.7 \pm 0.5$ & $1.6 \pm 0.5$ & $1.7 \pm 0.5$ & $1.8 \pm 0.6$ & 0.248 \\
\hline $\mathrm{Ca} \times \mathrm{P}$ product $\left(\mathrm{mg}^{2} / \mathrm{dL}^{2}\right)$ & $50.4 \pm 16.5$ & $47.2 \pm 13.8$ & $53.2 \pm 16.6$ & $56.8 \pm 19.5$ & 0.103 \\
\hline iPTH (pg/L) & $265.0(128.4,412.5)$ & $215.8(113.2,353.4)$ & $255.1(130.6,535.3)^{\mathrm{a}}$ & $327.7(126.4,610)^{\mathrm{a}}$ & $0.009^{*}$ \\
\hline $\mathrm{RRF}\left(\mathrm{mL} / \mathrm{min} \cdot 1.73 \mathrm{~m}^{2}\right)$ & $1.5(0.5,3.1)$ & $1.7(0.3,3.9)$ & $1.5(0.4,2.8)$ & $1.5(0.6,2.7)$ & 0.394 \\
\hline $\mathrm{KT} / \mathrm{V}$ & $2.2 \pm 0.5$ & $2.1 \pm 0.4$ & $2.2 \pm 0.6$ & $2.1 \pm 0.4$ & 0.403 \\
\hline
\end{tabular}

a: compared with no pruritus group, $\mathrm{p}<0.05$

b: compared with mild to moderate pruritus group, $\mathrm{p}<0.05$

PSQI: Pittsburgh Sleep Quality Index, BDI: Beck Depression Inventory, MIS: malnutrition inflammation score, CCI: Charlson Comorbidity Index, PCS: physical component score, MCS: mental component score, hsCRP: high-sensitivity C-reactive protein, Hb: hemoglobin, $\mathrm{Ca} \times \mathrm{P}$ product: calcium $\times$ phosphate product, iPTH: intact parathyroid hormone, BMI: body mass index, RRF: residue renal failure, BP: blood pressure

\#:1 Employed 2 Retired, unemployed, housewife

bance was an intermediate variable in the relationship between depressive symptoms and pruritus.

Our finding showed the severity of patient-reported pruritus and its relationship with PCS QoL scores. This finding was similar to what was reported by Curtin et al. (16), who described a significant inverse relationship between pruritus and PCS. While in this study, the effect of pruritus on the patient's physical quality of life disappeared in a multivariate analysis after adjustming for sleep quality, depression, and other confounders. Indeed, some studies reported that a higher degree of pruritus was more likely to correlate with a poorer mental and physical quality of life (8) and suggested that pruritus could have a negative impact on sleep quality and ultimately affect physical and mental functioning (22). Since depression and sleep quality were also the independent predictors of pruritus, we presumed that depression and sleep disorder might be the link between pruritus and PCS.

An interrelationship between pruritus, malnutrition, and inflammation had been demonstrated in the present study. MIS was used as a tool to assess the malnutritioninflammation status and a significant positive correlation between pruritus and MIS among the PD patients has been found. Moreover, the higher high-sensitivity C-reactive protein (hsCRP) level and MIS score that was observed in patients with severe pruritus in the present study was consistent with some studies that showed higher serum C-reactive protein and lower serum albumin levels were observed in patients with severe pruritus compared to those without it (6). Inflammation may play a role in the development of pruritus among HD patients. Some studies have reported HD patients with severe pruritus tended to have increased inflammatory parameters $(6,23)$. However, no inflammatory marker was independently associated with pruritus after correcting for potential confounders in the present study. Since our previous study demonstrated a strong association between MIS and poor sleep quality (11), sleep disorder may be the confounder for the relationship between malnutritioninflammation status and pruritus.

In this study, the $\mathrm{Kt} / \mathrm{v}$ values and residue renal failure (RRF) were not different between the patients with pruritus and the patients without pruritus. This result was consistent with the other previous studies which reported that the $\mathrm{Kt} / \mathrm{v}$ level and RRF were not associated with the prevalence and degree of pruritus in dialysis patients $(2,6) . \mathrm{Kt} / \mathrm{v}$ is a repre- 
Table 2. Multi-variable-adjusted Logistic Regression Analysis for Pruritus (VAS $\geq 1$ ), Depression (BDI Score $\geq 14$ ) and Sleep Disorder (PSQI $\geq 5$ ) in 362 CAPD Patients.

\begin{tabular}{|c|c|c|c|c|c|c|c|c|c|}
\hline \multicolumn{4}{|c|}{ Pruritus $^{a}$} & \multicolumn{3}{|c|}{ Sleep disorder ${ }^{b}$} & \multicolumn{3}{|c|}{ Depression $^{\mathrm{c}}$} \\
\hline Variables & OR & $95 \% \mathrm{CI}$ & $\mathrm{p}$ value & OR & $95 \% \mathrm{CI}$ & $\mathrm{p}$ value & OR & $95 \% \mathrm{CI}$ & $\mathrm{p}$ value \\
\hline Age & 1.01 & $0.96-1.05$ & 0.732 & 1.02 & $0.99-1.06$ & 0.132 & 1.04 & $0.99-1.04$ & 0.275 \\
\hline Female & 0.85 & $0.65-1.62$ & 0.336 & 1.27 & $0.57-2.84$ & 0.132 & 6.46 & $1.48-28.03$ & $0.013^{*}$ \\
\hline Vintage & 1.04 & $1.05-2.57$ & $0.004 *$ & 1.01 & $0.98-1.05$ & 0.395 & 0.96 & $0.93-1.86$ & 0.834 \\
\hline Diabetes & 1.52 & $0.43-5.28$ & 0.525 & 2.11 & $0.72-6.21$ & 0.173 & 1.83 & $1.27-4.87$ & $0.004 *$ \\
\hline $\mathrm{CCI}$ & 1.28 & $0.73-1.82$ & 0.592 & 1.07 & $0.83-1.27$ & 0.065 & 1.65 & $0.98-4.67$ & 0.745 \\
\hline VAS & --- & --- & --- & 1.65 & $1.13-2.42$ & $0.01 *$ & 0.96 & $0.75-1.33$ & 0.897 \\
\hline PSQI & 1.31 & $1.08-2.38$ & $0.001 *$ & --- & --- & --- & 1.17 & $1.03-1.34$ & $0.018^{*}$ \\
\hline BDI & 1.43 & $1.05-1.52$ & $0.002 *$ & 1.07 & $1.01-1.13$ & $0.024 *$ & --- & -- & -- \\
\hline MIS & 0.60 & $0.46-1.03$ & 0.328 & 1.19 & $1.01-1.87$ & $0.014 *$ & 1.04 & $0.99-1.07$ & 0.423 \\
\hline iPTH & 1.32 & $1.10-1.83$ & $0.014 *$ & 1.26 & $1.09-2.33$ & 0.41 & 1.27 & $0.87-2.14$ & 0.611 \\
\hline $\mathrm{Ca} \times \mathrm{P}$ & 0.78 & $0.36-1.93$ & 0.302 & 0.93 & $0.87-1.54$ & 0.259 & 0.86 & $0.69-1.08$ & 0.707 \\
\hline RRF & 0.31 & $0.29-0.78$ & 0.509 & 0.84 & $0.76-1.02$ & 0.837 & 0.79 & $0.65-1.00$ & 0.965 \\
\hline Season & 0.23 & $0.18-1.28$ & 0.214 & --- & --- & --- & --- & --- & --- \\
\hline
\end{tabular}

Notes: $* \mathrm{p}<0.05 ; * * \mathrm{p}<0.01$

a. dependent variable: Pruritus(VAS $\geq 1$ ), b. dependent variable: Sleep disorder (PSQI $\geq 5$ ), c. dependent variable: Depression (BDI score $\geq 14$ ), CCI: Charlson Comorbidity Index, VAS: visual analogue scale, PSQI: Pittsburgh sleep quality, BDI: Beck Depression Inventory score, MIS: malnutrition inflammation score, $\mathrm{iPTH}$ : intact parathyroid hormone

sentative of clearance of small molecular solute. Similar to previous studies, the present study has shown that the accumulation of middle molecular uremic toxins, such as iPTH, was significantly correlated to uraemic pruritus in CAPD patients. Regretfully, the data of another representative of middle molecular toxin of Beta-2 micrglobin was not collected in the present study. Therefore, further study is needed to testify the relation between pruritus and the removal of middle molecule uremic toxin. Theoretically, RRF loss should contribute to the uremic pruritus, since the clearance of the uremic toxins decreases along with the loss of RRF. However, the RRF did not show any relationship with pruritus in either the univariate or the multivariate analysis in the present study. We assume this result may imply that the gathering of the pruritus-causing toxins might be correlated with the regulation disorders of internal environment. Therefore, the relationship between RRF and accumulation of middle molecular uremic toxins are far more complex than our present understanding and further study is warranted in future.

The present study showed a close relationship between iPTH and pruritus and did not support any strong associations of pruritus with serum calcium and phosphorus levels. The relationship between hyperparathyroidism and pruritus was contraindicated. Intact PTH was initially suspected of being involved in causing pruritus in the present study and in another study (22), but was subsequently found not to be the main cause $(5,24)$. Indeed, a previous study has shown a greater deposit of calcium in the deepest layer of the dermis of HD patients with more severe pruritus (25). Although calcium and phosphate were not found to be associated with pruritus in the present study, an elevated calcium $\times$ phosphate product has been associated with uremic pruritus (26), Considering the close relationship between calcium $\times$ phosphate product and hyperparathyroidism, this study cannot rule out the role of disturbances of serum calcium and phos- phorus levels in the pathogenesis of pruritus among PD patients. In addition, we also observed that patients with a longer PD duration would experience severe pruritus, therefore more attention should be paid to the pruritus symptoms of those patient populations who have received longer PD treatment.

\section{Limitation}

Some limitations of this study should be noted. This was a cross-sectional and single-center study. The prevalence of pruritus was multifactorial and was influenced by center bias. Although confounders have been adjusted by statistical efforts, the effect of treatment for pruritus was not included in the model because we did not have enough data for analysis, which may cause bias in analyzing for the risk factors for pruritus. Moreover, it cannot be completely precluded whether the associations are causal or if any residual confounder remains. In addition, it was not possible to determine whether pruritus anteceded the appearance of depression symptoms and sleep problems among PD patients. Further study is necessary to explore the complex relationships involving pruritus, sleep problems, and depression, in order to develop a better understanding of their consequences and pathogenesis in CAPD patients.

\section{Conclusion}

Our study clearly demonstrated strong correlations existing between pruritus, sleep quality, and depressive symptoms in CAPD patients. After learning about the high prevalence of pruritus and related factors in the PD patients, we suggested effective interventions to alleviate depression and sleep disturbance symptoms, which may be beneficial for uraemic pruritus in CAPD patients. 
Table 3. Multivariate Linear Regression Models for Predictors of Quality of Life.

\begin{tabular}{|c|c|c|c|c|c|c|}
\hline \multirow[b]{2}{*}{ Variable } & \multicolumn{3}{|c|}{ PCS $^{d}$} & \multicolumn{3}{|c|}{ MCS $^{\mathrm{e}}$} \\
\hline & Beta & $\mathrm{p}$ value & $95 \% \mathrm{CI}$ for $\mathrm{B}$ & Beta & $\mathrm{p}$ value & $95 \%$ CI for B \\
\hline Age & -0.25 & $0.001^{* *}$ & $-0.28-0.10$ & 0.18 & $0.024^{*}$ & $0.12-1.05$ \\
\hline Vintage & -0.16 & 0.122 & --- & -0.02 & 0.698 & --- \\
\hline Diabetes & 0.09 & 0.182 & --- & -0.02 & 0.793 & --- \\
\hline PSQI & --- & 0.173 & --- & -0.27 & $0.001 * *$ & $-1.24--0.15$ \\
\hline BDI & -0.31 & $0.001 * *$ & $-1.02--0.15$ & -0.40 & $0.001 * *$ & $-0.59--0.14$ \\
\hline VAS & --- & 0.378 & --- - & ---- & 0.924 & --- - \\
\hline MIS & -0.26 & $0.001 * *$ & $-0.82--0.14$ & ---- & 0.529 & ---- \\
\hline CCI & -0.26 & 0.073 & --- & -0.03 & 0.351 & --- \\
\hline $\mathrm{Ca} \times \mathrm{P}$ & -0.15 & $0.019^{*}$ & $-0.13-0.01$ & --- & 0.981 & --- \\
\hline \multicolumn{7}{|c|}{$\begin{array}{l}\text { Notes: }{ }^{*} \mathrm{p}<0.05 ; * * \mathrm{p}<0.01 \\
\mathrm{~d} \text {. dependent variable: PCS, e. dependent variable: MCS, PCS : physical component } \\
\text { score, MCS: mental component score, PSQI: Pittsburgh Sleep Quality, BDI: Beck } \\
\text { Depression Inventory, VAS: visual analogue scale, MIS: malnutrition inflammation } \\
\text { score, CCI: Charlson Comorbidity Index, Ca } \times \text { P product: calcium } \times \text { phosphate } \\
\text { product }\end{array}$} \\
\hline
\end{tabular}

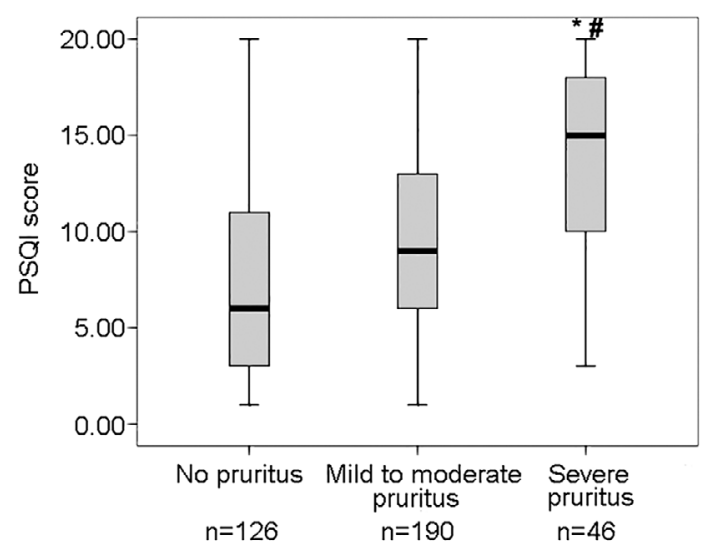

Figure 1. The patients with severe pruritus had the highest Pittsburgh Sleep Quality (PSQI) score $(12.3 \pm 5.6$ vs. $9.3 \pm 4.2$ vs. $7.7 \pm 5.3, p<0.001)$ among the three groups. *: Compared with mild to moderate pruritus group, $\mathbf{p}<\mathbf{0 . 0 5}$, \#: Compared with no pruritus group, $\mathrm{p}<0.05$

The authors state that they have no Conflict of Interest (COI).

\section{Financial Support}

This study was supported by The National Key Technology Research and Development Program of the Ministry of Science and Technology of China (Grant No. 2011BAI10B05), and by The Key Clinical Discipline Program of the Ministry of Health, China (Grant No [2010] 439).

\section{Acknowledgement}

We would like to thank the patients and personnel involved in this study.

\section{References}

1. Szepietowski JC, Balaskas E, Taube KM, Taberly A, Dupuy P; Uraemic Xerosis Working Group. Quality of life in patients with uraemic xerosis and pruritus. Acta Derm Venereol 91: 313-317, 2011.

2. Mathur VS, Lindberg J, Germain M, et al; ITCH National Regis-

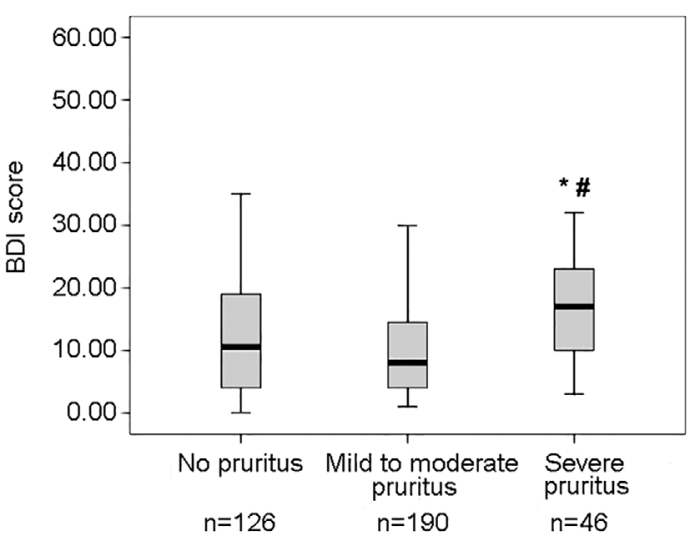

Figure 2. The patients with severe pruritus had the highest Beck Depression Inventory (BDI) score $[17(6,22)$ vs. $8(4,13)$ vs. $10.5(4,19.3), p=0.003$ ] among the three groups. *: Compared with mild to moderate pruritus group, $\mathbf{p}<0.05$, \#: Compared with no pruritus group, $\mathbf{p}<0.05$

try Investigators. A longitudinal study of uremic pruritus in hemodialysis patients. Clin J Am Soc Nephrol 5: 1410-1419, 2010.

3. Melo NC, Elias RM, Castro MC, Romao JE Jr, Abensur H. Pruritus in hemodialysis patients: the problem remains. Hemodial Int 13: 38-42, 2009.

4. Ko MJ, Wu HY, Chen HY, et al. Uremic pruritus, dialysis adequacy, and metabolic profiles in hemodialysis patients: a prospective 5-year cohort study. PLoS One 8: e71404, 2013.

5. Noordzij M, Boeschoten EW, Bos WJ, et al; NECOSAD Study Group. Disturbed mineral metabolism is associated with muscle and skin complaints in a prospective cohort of dialysis patients. Nephrol Dial Transplant 22: 2944-2949, 2007.

6. Chiu YL, Chen HY, Chuang YF, et al. Association of uraemic pruritus with inflammation and hepatitis infection in haemodialysis patients. Nephrol Dial Transplant 23: 3685-3689, 2008.

7. Bolier R, Oude Elferink RP, Beuers U. Advances in pathogenesis and treatment of pruritus. Clin Liver Dis 17: 319-329, 2013.

8. Pisoni RL, Wikström B, Elder SJ, et al. Pruritus in haemodialysis patients: International results from the Dialysis Outcomes and Practice Patterns Study (DOPPS). Nephrol Dial Transplant 21: 3495-3505, 2006.

9. Lopes GB, Nogueira FC, de Souza MR, et al. Assessment of the 
psychological burden associated with pruritus in hemodialysis patients using the kidney disease quality of life short form. Qual Life Res 21: 603-612, 2011.

10. Kimata N, Fuller DS, Saito A, et al. Pruritus in hemodialysis patients: Results from the Japanese Dialysis Outcomes and Practice Patterns Study (JDOPPS). Hemodial Int 18: 657-667, 2014.

11. Li J, Guo Q, Ye X, et al. Prevalence and risk factors of sleep disturbance in continuous ambulatory peritoneal dialysis patients in Guangzhou, southern China. Int Urol Nephrol 44: 929-936, 2012.

12. Peng YS, Chiang CK, Hung KY, et al. Comparison of selfreported health-related quality of life between Taiwan hemodialysis and peritoneal dialysis patients: a multi-center collaborative study. Qual Life Res 20: 399-405, 2011.

13. Ye XQ, Chen WQ, Lin JX, et al. Effect of social support on psychological-stress-induced anxiety and depressive symptoms in patients receiving peritoneal dialysis. J Psychosom Res 65: 157164, 2008.

14. Yong DS, Kwok AO, Wong DM, Suen MH, Chen WT, Tse DM. Symptom burden and quality of life in end-stage renal disease: a study of 179 patients on dialysis and palliative care. Palliat Med 23: 111-119, 2009.

15. Snit M, Gawlik R, Łącka-Gaździk B, et al. Substance $P$ and intensity of pruritus in hemodialysis and peritoneal dialysis patients. Med Sci Monit 19: 723-732, 2013.

16. Wikstrom B. Itchy skin--a clinical problem for haemodialysis patients. Nephrol Dial Transplant 22 (Suppl 5): v3-v7, 2007.

17. Patel T, Ishiuji Y, Yosipovitch G. Nocturnal itch: why do we itch at night? Acta Derm Venereol 87: 295-298, 2007.

18. Chou KL. Reciprocal relationship between pain and depression in older adults: evidence from the English Longitudinal Study of Ageing. J Affect Disord 102: 115-123, 2007.

19. Yamamoto Y, Hayashino Y, Yamazaki S, et al; J-DOPPS Research Group. Depressive symptoms predict the future risk of severe pruritus in haemodialysis patients: Japan Dialysis Outcomes and Practice Patterns Study. Br J Dermatol 161: 384-389, 2009.

20. Yang JY, Huang JW, Peng YS, et al. Quality of sleep and psychosocial factors for patients undergoing peritoneal dialysis. Perit Dial Int 27: 675-680, 2007.

21. Guney I, Biyik M, Yeksan M, et al. Sleep quality and depression in peritoneal dialysis patients. Ren Fail 30: 1017-1022, 2008.

22. Aleckovic-Halilovic M, Mesic E, Jasarevic A, Halilbasic A. Quiz page August 2014: Pruritus in a dialysis patient. Mycosis fungoides (folliculotropic variant) in a dialysis patient, with rare presentation of persistent generalized pruritus with characteristics of uremic pruritus without skin lesions. Am J Kidney Dis 64: xviii-xxii, 2014.

23. Kimmel M, Alscher DM, Dunst R, et al. The role of microinflammation in the pathogenesis of uraemic pruritus in haemodialysis patients. Nephrol Dial Transplant 21: 749-755, 2006.

24. Duque MI, Thevarajah S, Chan YH, Tuttle AB, Freedman BI, Yosipovitch G. Uremic pruritus is associated with higher $\mathrm{kt} / \mathrm{V}$ and serum calcium concentration. Clin Nephrol 66: 184-191, 2006.

25. Momose A, Kudo S, Sato M, et al. Calcium ions are abnormally distributed in the skin of haemodialysis patients with uraemic pruritus. Nephrol Dial Transplant 19: 2061-2066, 2004.

26. Yong AS, Lee KY. Uremic pruritus is improved by gabapentin. Int J Dermatol 53: e404-e405, 2014.

(C) 2015 The Japanese Society of Internal Medicine http://www.naika.or.jp/imonline/index.html 